\title{
Tomato $D C L 2 b$ is required for the biosynthesis of 22-nt small RNAs, the resulting secondary siRNAs, and the host defense against ToMV
}

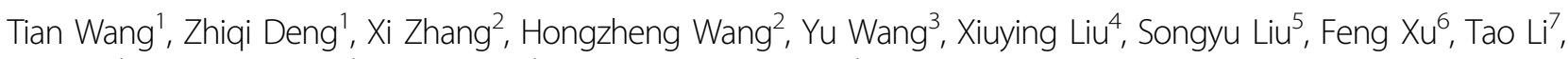 \\ Daqi Fu', Benzhong Zhu', Yunbo Luo' and Hongliang Zhu (i)
}

\begin{abstract}
The tomato encode four functional $D C L$ families, of which $D C L 2$ is poorly studied. Here, we generated loss-of-function mutants for a tomato $D C L 2$ gene, $d C l 2 b$, and we identified its major role in defending against tomato mosaic virus in relation to both natural and manual infections. Genome-wide small RNA expression profiling revealed that DCL2b was required for the processing 22-nt small RNAs, including a few species of miRNAs. Interestingly, these DCL2b-dependent 22-nt miRNAs functioned similarly to the DCL1-produced 22-nt miRNAs in Arabidopsis and could serve as triggers to generate a class of secondary siRNAs. In particular, the majority of secondary siRNAs were derived from plant defense genes when the plants were challenged with viruses. We also examined differentially expressed genes in $d c / 2 b$ through RNA-seq and observed that numerous genes were associated with mitochondrial metabolism and hormone signaling under virus-free conditions. Notably, when the loss-of-function $d c / 2 b$ mutant was challenged with tomato mosaic virus, a group of defense response genes was activated, whereas the genes related to lipid metabolism were suppressed. Together, our findings provided new insights into the roles of tomato DCL2b in small RNA biogenesis and in antiviral defense.
\end{abstract}

\section{Introduction}

RNA silencing plays key roles in regulating endogenous gene expression, suppressing transposon activity, silencing transgenes, responding to environmental stimuli and combatting viral infection ${ }^{1,2}$. Small RNAs (sRNAs), including miRNA and siRNA, are loaded into an Argonaute (AGO) effector protein to form RNA-induced silencing complexes to repress complementary target RNA at a posttranscriptional gene silencing (PTGS) level through cleavage and/or the inhibition of translation.

\footnotetext{
Correspondence: Hongliang Zhu (hlzhu@cau.edu.cn)

${ }^{1}$ College of Food Science and Nutritional Engineering, China Agricultural University, 100083 Beijing, China

${ }^{2}$ Key Laboratory of Horticultural Plant Biology, Ministry of Education, College of Horticulture and Forestry Sciences, Huazhong Agricultural University, 430070 Wuhan, China

Full list of author information is available at the end of the article.
}

RNA-induced silencing complexes can also repress gene expression at a transcriptional gene (TGS) silencing level ${ }^{3,4}$.

sRNAs are generated by $D C L$ proteins. In Arabidopsis, four $D C L$ family proteins have been found to cleave stemloop or double-stranded (ds) RNA precursors into miRNAs or siRNAs of specific sizes ${ }^{5}$. DCL1 primarily processes hairpin RNA into the 21-nt miRNA that is involved in PTGS ${ }^{6,7}$. However, DCL1 can also produce 22-nt miRNAs from bulged precursors that in turn lead to the production of secondary siRNAs. DCL2 is required for the biogenesis of 22-nt siRNAs from endogenous inverted repeat loci $^{8,9}$. DCL3 produces TGS-engaged 24-nt siRNAs from RNA-dependent RNA Polymerase 2 (RDR2)dependent dsRNAs ${ }^{4,10}$. DCL4 produces 21-nt siRNAs from RDR6-dependent dsRNA in the PTGS pathway ${ }^{11,12}$.

\section{(c) The Author(s) 2018}

(c) (i) Open Access This article is licensed under a Creative Commons Attribution 4.0 International License, which permits use, sharing, adaptation, distribution and reproduction in any medium or format, as long as you give appropriate credit to the original author(s) and the source, provide a link to the Creative Commons license, and indicate if changes were made. The images or other third party material in this article are included in the article's Creative Commons license, unless indicated otherwise in a credit line to the material. If material is not included in the article's Creative Commons license and your intended use is not permitted by statutory regulation or exceeds the permitted use, you will need to obtain permission directly from the copyright holder. To view a copy of this license, visit http://creativecommons.org/licenses/by/4.0/. 
In addition, DCL3 and DCL2 could also generate secondary siRNA (sec-siRNAs) from RDR6-dependent dsRNA in a $d c l 4$ mutant ${ }^{13}$.

Sec-siRNAs typically exhibit a phased pattern, and they have recently been shown to be derived from a large set of loci; they play important roles in plant development and disease resistance ${ }^{14,15}$. Normally, sec-siRNAs are processed from $3^{\prime}$ cleaved transcripts that are targeted by DCL1-generated 22-nt miRNA and trans-acting siRNA (tasiRNA) $)^{16,17}$, except for 21-nt miR390 ${ }^{18}$. The $3^{\prime}$ cleaved transcripts are amplified into dsRNAs by RDR6 and then cleaved by DCL4 into 21-nt 'head-to-tail'-phased, secsiRNAs ${ }^{14,18}$ or cleaved by DCL3 into 24 -nt sec-siRNA ${ }^{12}$. These sec-siRNAs are called phased siRNA (phasiRNA) if they are generated from coding transcripts, but they are known as tasiRNA if they come from noncoding transcripts. Many miRNAs could trigger the production of sec-siRNAs from various RNA transcripts, such as the pairs miR173 and TAS1/2, miR390 and TAS3, miR393 and TIR/AFB, miR7122 and PPR, and miR482 and NBS$L R R^{14,15}$.

Over the past few decades, the biological roles of $D C L 1$, $D C L 3$ and DCL4 have been well studied, and the DCL2 was considered as a substitute for DCL4 for defense against viruses ${ }^{19,20}$. Importantly, DCL2 plays a primary role in transgene silencing, especially in the sense transgene-induced silencing and transitivity of hairpininduced transgene silencing ${ }^{21,22}$. DCL2 is also engaged in plant development and systemic RNA silencing, and DCL4 attenuates systemic PTGS ${ }^{8,23,24}$. When the DCL4 function is impaired, endogenous genes (SMXL4 and SMXL5) are excessively silenced by 22 -nt siRNA that are produced from the DCL2- and RDR6-dependent transitive PTGS pathway. In wild type (WT) plants, DCL4 outcompetes $D C L 2$ for the same dsRNA templates, which prevents or limits the deleterious effects of this endogenous silencing by $D C L 2^{8}$. DCL2 can enhance the recruitment of RDR6 to target transcripts and the production of sec-siRNAs. The DCL2-produced 22-nt siRNA can also trigger the production of 21-nt sec-siRNA from target mRNA ${ }^{9,23,24}$. This type of siRNA amplification is essential for enhancing the PTGS efficiency. It is now proposed that there is a dualdefense strategy for plant viruses to break. DCL4 is the primary defender that attacks viruses in initially infected cells through virus-induced gene silencing in autonomous cells. Once the DCL4 activity is inhibited by the viral suppressors of RNA silencing, DCL2 and DCL2-processed/dependent siRNAs are required to trigger non-cell autonomous (virus-induced gene silencing) and protect the recipient cells from further invasion by plant viruses $^{23,24}$.

The tomato (Solanum lycopersicum) is the seventhmost important crop species and the second-most consumed vegetable in the world ${ }^{25}$. An analysis of tomato
$D C L 1$ and $D C L 3$-silencing mutants indicates that DCL1 produces canonical miRNAs and a few 21-nt siRNAs ${ }^{7}$, and DCL3 is involved in the biosynthesis of heterochromatic 24-nt siRNAs and long miRNAs ${ }^{10}$. DCL4 is required for the production of 21-nt tasiRNAs that in turn target the ARFs to alter tomato leaf development ${ }^{11}$. However, the functions of tomato DCL2 remain unknown. Previously, we cloned the full-length cDNA sequences of four tomato $D C L 2$ subfamily members, $D C L 2 a, D C L 2 b, D C L 2 c$, and $D C L 2 d$ for expression pattern analysis, and we showed that the $D C L 2 b$ expression is much higher than that of other $D C L 2 s$, implying its predominant role in biology ${ }^{26}$.

Here, we generated loss-of-function $d c l 2 b$ mutants using the CRISPR/Cas9 genome-editing system. The $d c l 2 b$ mutants did not show developmental defects under normal conditions. When infected by tomato mosaic virus (ToMV), however, the $d c l 2 b$ mutants displayed more severe developmental defects consisting of strange narrow patterns on the leaves, flowers and fruits compared to the WT. Even DCL4 was still functional, indicating that $D C L 2 b$ played a major role in the defense against ToMV. We performed genome-wide sRNA expression profiling and found that $D C L 2 b$ was required for the biogenesis of 22-nt sRNA, including some 22-nt miRNAs that would otherwise be produced by DCL1 in Arabidopsis, leading to sec-siRNA production. The RNA-seq analysis showed that numerous genes associated with mitochondrial metabolism and hormone signaling changed significantly under virus-free conditions. When the ToMV engaged in infection, the tomato plants activated the genes involved in a response pathway to the stimulus and suppressed a lipid metabolism pathway. Collectively, our results demonstrated that tomato $D C L 2 b$ played a critical role in antiviral defense by regulating the biogenesis of a group of 22-nt miRNAs and subsequently sec-siRNAs.

\section{Results}

Generation of $d c \mid 2 b$ mutants using the CRISPR/Cas9 geneediting system

To generate null alleles of loss-of-function mutants, we used the CRISPR/Cas9 gene-editing system to knock out $D C L 2 b$ in tomatoes. Four genomic sites were targeted for cleavage (Figure S1a), and then transgenic plants were genotyped through the direct sequencing of PCR products from genomic DNA flanking the target sites. The transgenic lines were grown in two greenhouses (I and II) under comparable cultivation environments. Two lines from each greenhouse carrying homozygous deletions of $5 \mathrm{bp}$ in Target1 were predicted to be null mutants and selected for further research (Figure S1b). The genome editing caused a premature stop codon to occur in the first conserved domain of the $D C L 2 b$ protein (Figure S1c). We next predicted there would be four off-target genes for each edited 
target in the tomato genome using CRISPR-GE ${ }^{27}$. No offtarget events were detected at any sites (Figure S2).

\section{$d c / 2 b$ mutants displayed different phenotypes in two greenhouses}

In greenhouse I, the tomato WT and $d c l 2 b$ mutants were grown normally without any visible differences (Fig. 1a). However, all the $d c l 2 b$ plants in greenhouse II displayed a strange morphological phenotype compared with the WT. The adult leaves were abnormally long, narrow, and twisted, and the secondary leaflets disappeared. A scanning electron microscope (SEM) analysis of these long leaflets showed that the cells were distinct in size and shape. They became long and columnar rather than irregular polygons (Fig. 1a). In addition to the abnormal leaf development, we observed a similar phenotype in the flowers and fruits of $d c l 2 b$ mutants from greenhouse I. The flowers had more spindly petals and sepals (Fig. 1b, c). We compared the fruits of the WT and $d c l 2 b$ mutant at three stages, at 25 days after pollination (DPA), 35 DPA and 45 DPA. The mutant fruits exhibited an elongated shape whereas the WT fruits were almost round (Fig. 1d). Notably, the fruits of $d c l 2 b$ had few welldeveloped seeds (Fig. 1d). Furthermore, the fruit setting ratio of the first three branches in the mutant was much lower (Fig. 1e), which might partially result from developmental defects in the stamen and pistil (Fig. 1b).

\section{Strange narrow pattern phenotype in the $d c / 2 b$ mutant resulting from ToMV infection}

The strange phenotype was reminiscent of plant symptoms that occur when a viral infection takes place ${ }^{28}$. We wondered whether the morphological phenotype of the $d c l 2 b$ mutants grown in greenhouse II were caused by plant virus(es). Since the deep sequencing of virus-derived small-interfering RNAs (vsiRNAs) has been shown to be an efficient approach for virus discovery in plants and animals $^{29}$, we conducted sRNA-seq with total RNA prepared from the following samples: normal adult leaves from the WT and $d c l 2 b$ from greenhouse I, and normal adult leaves from the WT and "shoestring-like" adult leaves of $d c l 2 b$ from greenhouse II. More than 14 million clean reads ranging from 18 to $26 \mathrm{nt}$ were obtained. Notably, the tomato genome mapping ratios of the WT and $d c l 2 b$ from greenhouse II were obviously lower than those of greenhouse I (Figure S3), suggesting that part of the sequence reads might map to genomes other than that of the tomato. Next, we aligned all the cleaned sRNA-seq reads to 8605 viral genome sequences that were mined from the NCBI (https://www.ncbi.nlm.nih.gov/genome/ viruses/). Less than $0.01 \%$ of the reads from the WT and $d c l 2 b$ from greenhouse I could be mapped to virus genomes. However, $12.5-35.7 \%$ of the reads from greenhouse II matched viral genomes (Fig. 2a).
We analyzed the size distribution of vsiRNAs in the WT and $d c l 2 b$ mutant from greenhouse II and found that more than half of these reads reached 21-nt (Fig. 2b). This result was consistent with previous studies in which the majority of vsiRNAs are 21-nt long in virus-infected plants $^{20,30,31}$. Among the vsiRNAs, over $90 \%$ of the reads matched to ToMV, whereas $3.8 \%$ and $1.5 \%$ belonged to tomato mottle mosaic virus (ToMMV) and tomato brown rugose fruit virus (ToBRFV), respectively, with residue reads aligning to another 8000 viruses (Fig. 2c). The three species are positive-sense single-strand RNA (ssRNA) viruses that belong to the Tobamovirus genus ${ }^{32}$.

To examine whether the $d c l 2 b$ mutant was infected with a single ToMV virus or multi-viruses, we checked the distribution of vsiRNAs against 34 total types of tobamovirus RNA genomes from the NCBI GenBank database. The protein sequences of replicases from 34 viruses were aligned and an unrooted neighbor-joining tree was constructed (Fig. 2d). It was obvious that three tomato viruses, ToMV, ToMMV, and ToBRFV, had closer evolutionary relationships. Furthermore, compared with ToMMV and ToBRFV, the vsiRNAs were distributed over the entire ToMV. The few vsiRNA-matched loci in ToMMV and ToBRFV most likely resulted from the high sequence similarity with ToMV. Collectively, these results suggested that the WT and $d c l 2 b$ from greenhouse II were both infected naturally by ToMV. This conclusion was further validated by reverse transcription-PCR (RT-PCR), which showed that a ToMV-specific fragment was detected in the WT and $d c l 2 b$ from greenhouse II, but not in plants from greenhouse I (Fig. 2e). Taken together, these data indicated that the loss of tomato DCL2b would increase a plant's susceptibility to ToMV infection in a natural environment.

To validate the result above, we challenged the tomato WT and $d c l 2 b$ mutants at 2-, 3-, and 4-week stages in a virus-free chamber with ToMV. Again, the $d c l 2 b$ mutants infected by the virus all displayed a strange narrow phenotype while the WT grew normally (Figure S4). This result was fully reproducible in three independent experiments. In addition, an RNA blot showed that the viral RNA accumulation was clearly higher in $d c l 2 b$ than in the WT plants (Figure S4b). Altogether, the developmental defects observed in the $d c l 2 b$ mutant in both the greenhouse and the controlled growth chamber did result from ToMV infection, a further indication that our analysis of datasets collected from naturally and unbiased infection plants are physiologically relevant.

\section{DCL2b was required for the biogenesis of 22-nt sRNA}

To investigate the functions of $D C L 2 b$, we removed the vsiRNAs and focused on endogenous tomato sRNA data for further analysis. Genome-wide profiling by sRNA-seq showed that the tomato sRNAs were not evenly 


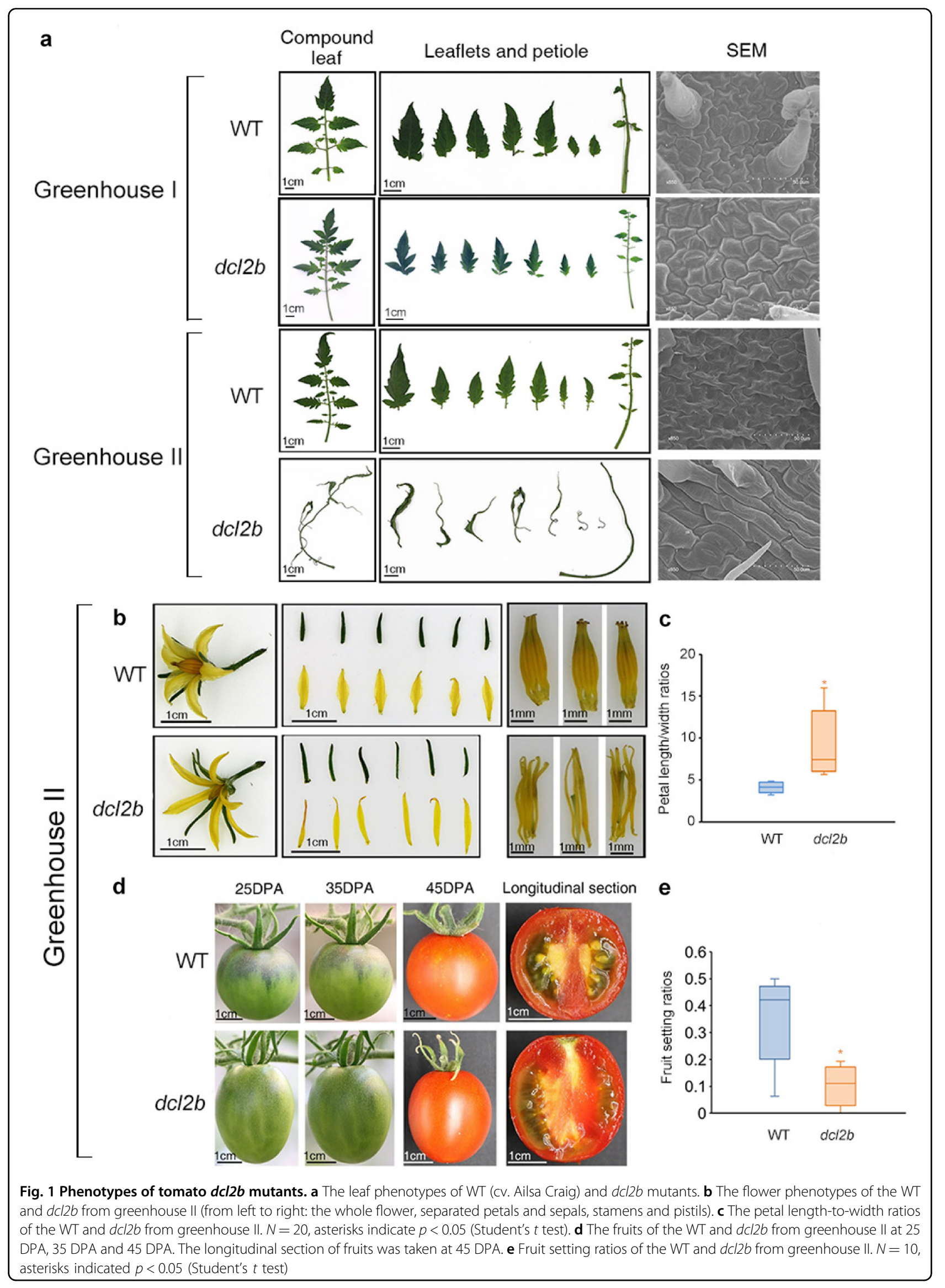




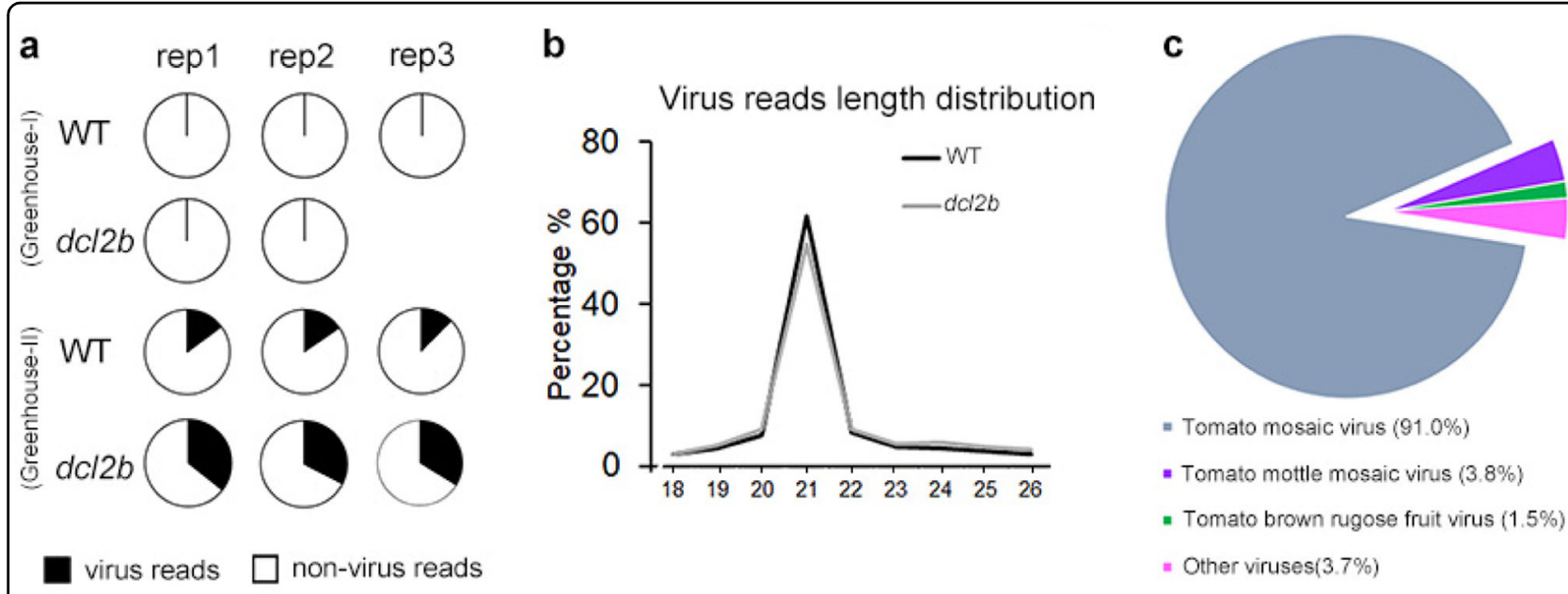

d
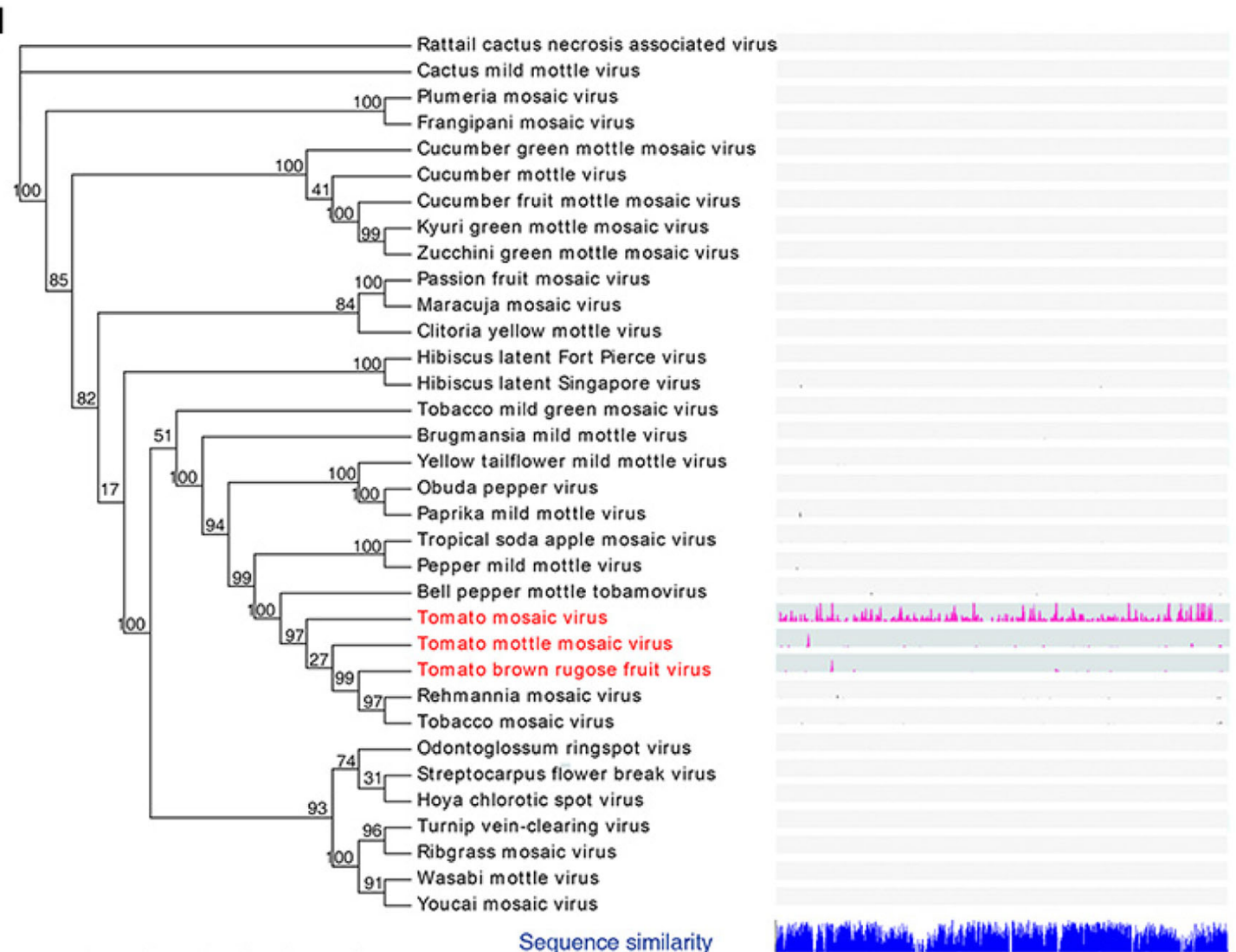

e Sequence similarity

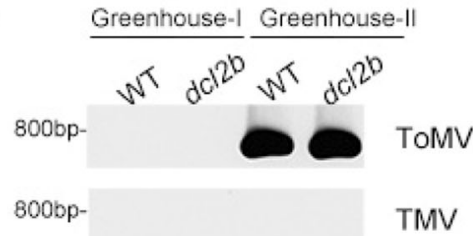

Fig. 2 sRNA-seq revealed natural ToMV infections in plants. a The percentage of virus-derived reads in the WT and $d c \mid 2 b$ samples. $\mathbf{b}$ The length distribution of virus-derived total reads in the WT and $d c 12 b$ from greenhouse II. c The distribution patterns of ToMV, TMMV, TBRFV, and other viruses. d Phylogenetic analyses of 34 Tobamoviruses. A phylogenetic analysis is shown in the left panel. The three viruses analyzed in the manuscript are marked in red. The genome-wide distributions of virus-derived reads are displayed on the right. e An RT-PCR analysis of specific fragments of ToMV and tobacco mosaic virus (TMV) 


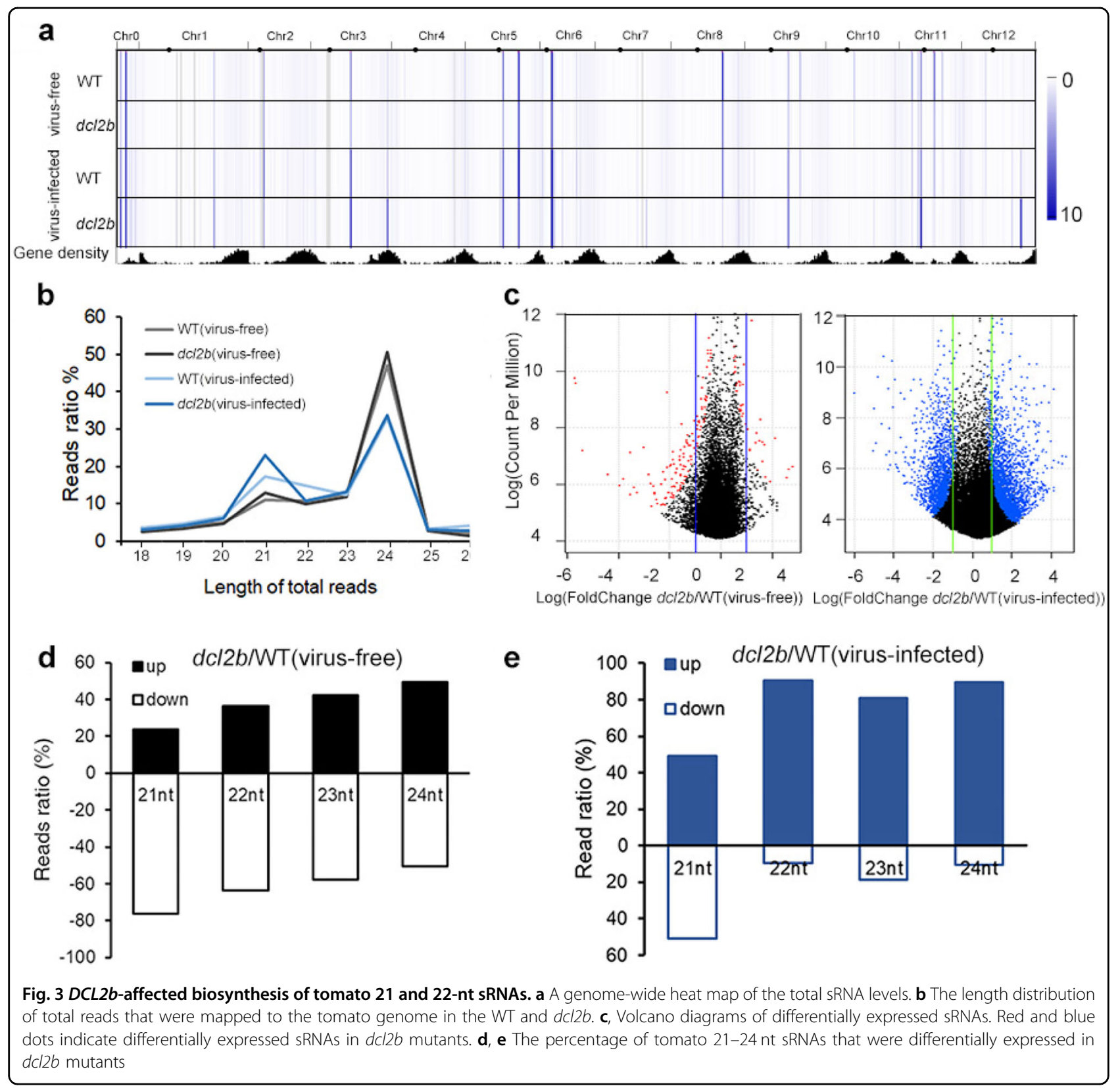

distributed across the chromosomes of the WT and mutants (Fig. 3a). Unlike the tomato long non-coding $\mathrm{RNAs}^{33}$, the sRNAs showed higher densities in the pericentromeric heterochromatin regions than in the euchromatin (Fig. 3a). Furthermore, the knockout of $D C L 2 b$ did not influence the overall sRNA distribution (Fig. 3a). Since DCL2 was previously reported to process dsRNA into 22-nt sRNA ${ }^{22}$, we counted the reads of each sRNA species and found that 22-nt sRNA did not experience a significant decrease in the virus-free $d c l 2 b$ mutant (Fig. 3b). Compared with plants grown in greenhouse I, the populations of 21-nt sRNAs were markedly enhanced in the WT and $d c l 2 b$ from greenhouse II (Fig. 3b). These results were consistent with the previous findings in which parts of these 21-nt sRNAs were novel siRNAs induced by viral infection, and they were designated as virus-activated siRNAs (vasiRNAs) ${ }^{34}$.

We then compared the differentially expressed endogenous sRNAs (DE sRNAs) in two groups. Compared with the WT, 750 sRNAs showed significant change in the $d c l 2 b$ mutant. Among them, 242 (32.3\%) were upregulated and $508(67.7 \%)$ sRNAs were downregulated (Fig. 3c). Under viral infection, the number of DE sRNAs rose to 4188 , for 3130 (74.7\%) increased and 1058 (25.3\%) 
decreased, respectively. These results suggested that the presence of the virus influenced large numbers of sRNAs. To illustrate the role of $D C L 2 b$ in the accumulation of these sRNAs, we separated 21, 22, 23, and 24-nt DE sRNAs and calculated their respective up- and downregulation proportions. Intriguingly, almost $65 \%$ of the 21 - and 22-nt DE sRNAs were decreased in the $d c l 2 b$ mutant whereas 23- and 24-nt sRNAs showed comparable bilateral changes (Fig. 3d). It has been known that 22-nt sRNAs can trigger the biogenesis of 21-nt sec-siRNAs that are typically produced by $D C L 4$ in plants ${ }^{15,16}$. Here, we tended to interpret that the production of 22-nt triggers was largely affected in the $d c l 2 b$ mutant, leading to a compromise in the generation of 21-nt sec-siRNAs under virus-free conditions. However, this scenario was not observed under the virus-infection condition since an overwhelming number of DE sRNAs were produced in the absence of $D C L 2 b$ (Fig. 3e).

\section{DCL2b affected miRNA accumulation and sec-siRNA production}

Sec-siRNAs can be triggered by 22 -nt miRNA. We next examined the impact of tomato $D C L 2 b$ in the regulation of miRNA expression. To this end, we calculated the enrichment levels of 110 mature tomato miRNAs from miRBase. We first examined the miRNA changes under the virus-free condition. Compared with the WT, only five miRNAs exhibited significant changes in the $d c l 2 b$ mutant (Fig. 4a). Notably, the expression levels of 21-nt miR399 and 22-nt miR6026 were reduced. A legitimate target site for miR6026 was predicted in the $5^{\prime}$ UTR region of $D C L 2 a, 2 b$, and $2 d$, raising the possibility that miR6026 might act as a trigger for sec-siRNA biogenesis from the three DCL2 genes ${ }^{7}$. In the virus-infected $d c l 2 b$ mutant, the number of DE miRNAs was elevated to 31, and the expression of DE miRNAs was easily validated by a small RNA blot analysis (Fig. 4b). Importantly, 9 of the $31 \mathrm{DE}$ miRNAs reached 22-nt (Fig. 4c). Interestingly, we aligned six 22-nt DE miRNA sequences and found that they all had a $\mathrm{U}$ in the $5^{\prime}$ position and three miRNAs had a $\mathrm{C}$ at the $3^{\prime}$ terminal nucleotide (Fig. $4 \mathrm{~d}$ ), indicating that these miRNAs were likely associated with the initiation of secsiRNA production ${ }^{16}$.

To investigate whether $D C L 2 b$ contributed to the secsiRNA production through 22-nt miRNAs, we aligned all the sRNAs measuring 18-26-nt to tomato transcripts (genome annotation ITAG3.2), revealing perfect matches using Bowtie and an in-house Perl program. We named the transcripts that served as the precursors of secsiRNAs as templates, and then we extracted the templates that contained less than half the reads of sec-siRNAs generated in $d c l 2 b$ mutants compared with their own WT controls. Here, we predicted 96 and 197 potential templates in virus-free and virus-infected $d c l 2 b$, respectively, with 17 overlaps (Fig. 4e and Table S1). In the previous studies, DCL4, together with AGO1, AGO4, AGO7, SUPPRESSOR OF GENE SILENCING3 (SGS3), RDR6, and DOUBLE-STRANDED RNA BINDING FACTOR 4 (DRB4), were found to participate in different steps of secsiRNA production ${ }^{15,35}$. To investigate if these above genes had any impact on the sec-siRNA production from the above filtered templates, we measured their abundance according to RNA-seq data that were obtained from the same sets of samples for the sRNA-seq above. We obtained 20 million clean reads from RNA-seq, over $80 \%$ of which could align to the tomato genome (with two mismatches) (Figure S3). We found that none of these sec-siRNA-related genes displayed obvious changes in transcription when $D C L 2 b$ was knocked out (Figure S5a). Thus, the differential accumulation of sec-siRNAs is unlikely caused through the indirect impact of these secsiRNA-related genes.

In the virus-free WT and $d c l 2 b$ mutant, we hypothesized that the 22-nt miR6026 might lead to the formation of sec-siRNA from targeted mRNAs such as DCL2a, $2 b$, and $2 d$ (Figure S5b). The expression level of miR6026 in the $d c l 2 b$ mutant decreased significantly (Fig. $4 \mathrm{~b}$ and Figure S5c). The sec-siRNA abundance from three DCL2 transcripts was reduced two- to seven-fold (Fig. 5a and Figure S5d). Intriguingly, over $70 \%$ of the total sec-siRNAs measured 21-nt (Fig. 5b), suggesting that DCL4 might play a major role in 21-nt sec-siRNAs biosynthesis from the DCL2 transcripts. It is noteworthy that the formation of 22-nt sec-siRNA was suppressed in the $d c l 2 b$ mutant, suggesting that $D C L 2 b$ is also involved in the biogenesis of 22-nt sec-siRNAs (Fig. 5b). Next, we used Integrative Genomics Viewer (IGV) to illustrate the above results. Normalized sRNAs were mapped to three DCL2 loci, and the IGV screenshots clearly showed that sec-siRNAs were produced in a phased pattern of each locus (Fig. 5a). We could also note that the sec-siRNAs were less abundant in the $d c l 2 b$ mutant (Fig. 5a). The miR6026 expression did not change significantly in the virus-infected $d c l 2 b$ mutant (Figure S5c). As a consequence, the abundance variations in DCL2-derived sec-siRNAs were not as obvious as those in the virus-free samples (Fig. $5 \mathrm{a}$ and Figure S5d).

In contrast to virus-free plants, more 22-nt miRNAs experienced significant decreases in the virus-infected $d c l 2 b$ mutant (Fig. 4a, c). We selected three downregulated ones for analysis to find if they acted as triggers of sec-siRNAs. Notably, three, four, and two genes had target loci for miR6027, miR482b, and miR482e, respectively, all of which are disease-related genes. The sec-siRNAs generated from these templates were remarkably reduced in the tomato $d c l 2 b$ mutant (Figure S5e). The IGV screenshots also illustrated the phased patterns of sec-siRNAs (Fig. 5c), indicating that the three 


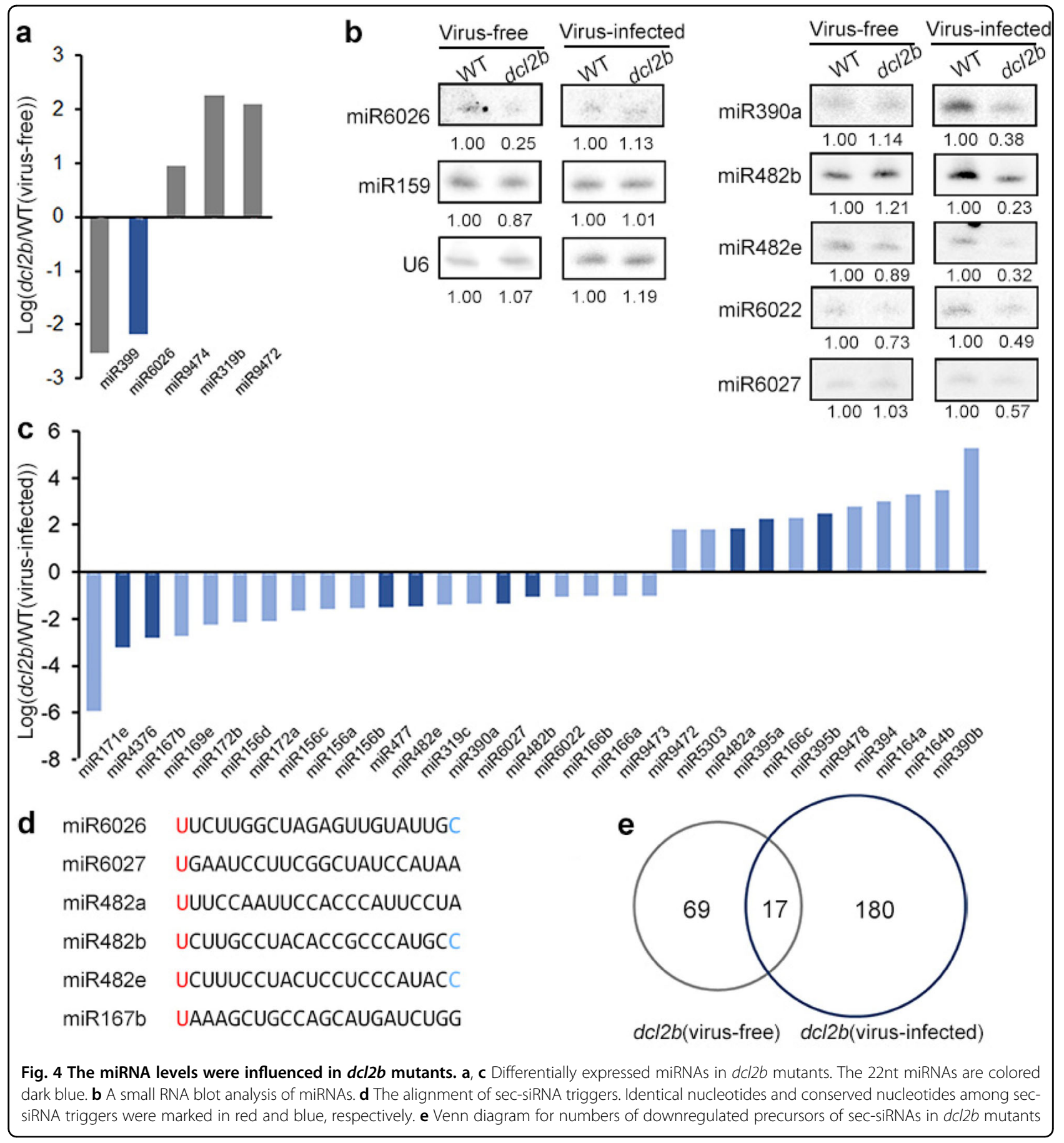

miRNAs caused target slicing and phased siRNA production. Furthermore, when we checked the annotations of the sec-siRNAs precursor from $d c l 2 b$, we found 66 out of 197 precursors belonged to the "disease resistant" category (Table S1), revealing $D C L 2 b$ 's critical function in the sec-siRNA biogenesis after ToMV infection. From the above results, we could conclude that $D C L 2 b$ plays an important role in sec-siRNA production. When tomato plants are infected by ToMV, $D C L 2 b$ plants have an even broader impact on disease-resistant genederived sec-siRNAs, at least partially through their 22-nt sRNA triggers.

\section{Function analysis of $D C L 2 b$-activated and -repressed genes}

To further investigate the function of tomato DCL2b, we examined the global expression profiles of the WT and 

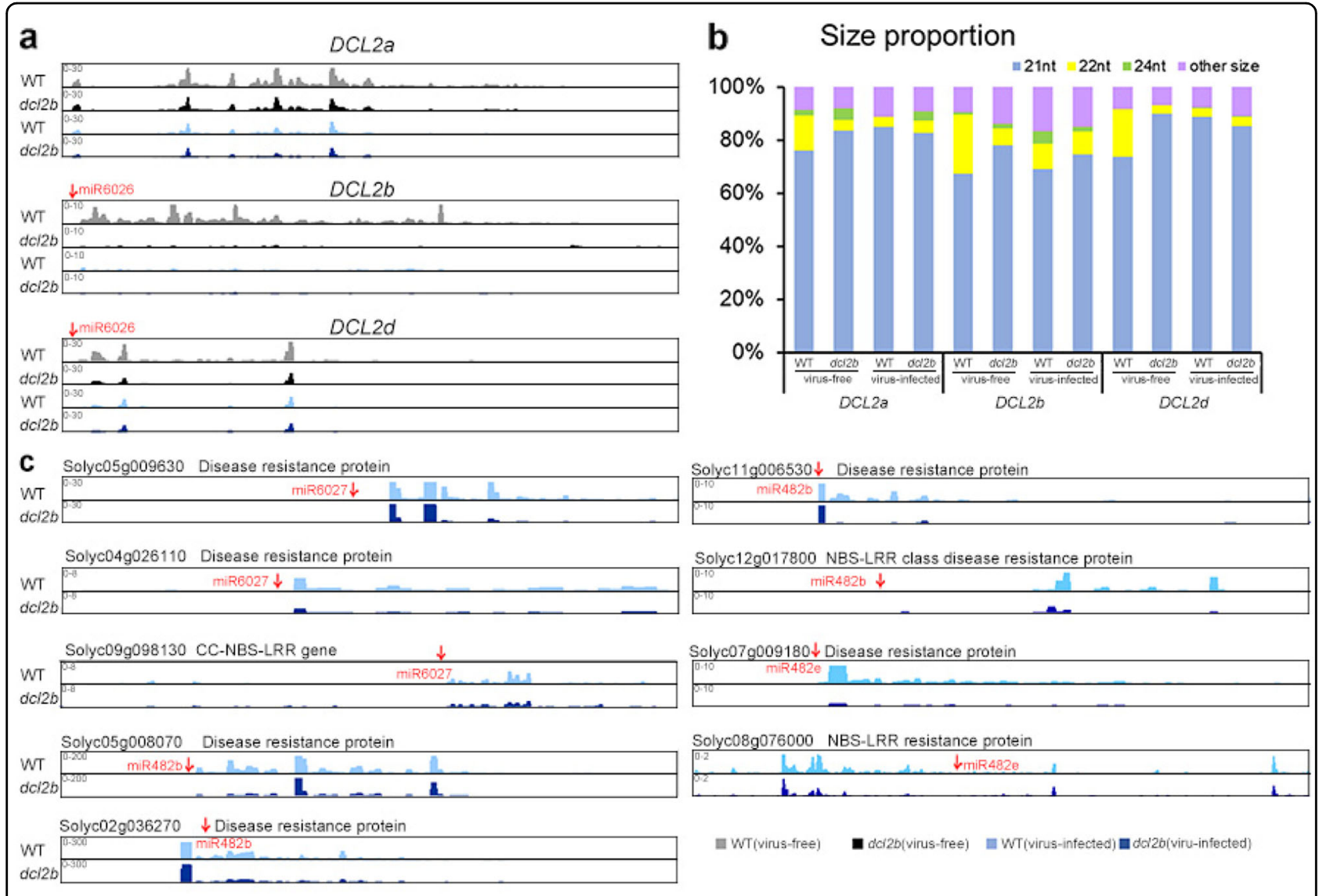

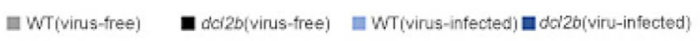

Fig. 5 Sec-siRNAs triggered by 22-nt miRNAs. a The sec-siRNA levels of DCL2a, $2 b$ and $2 d$ are shown with screenshots from Integrative Genomics Viewer (IGV). For the display, the three biological replicates of each sample were merged. Red arrows show the positions of sites complementary to miR6026. The horizontal axes represent the sRNA reads per million (RPM). $\mathbf{b}$ The size distribution of sec-siRNAs that are produced from DCL2a, $2 b$ and $2 d$. c The sec-siRNA levels of nine genes triggered by miR6027, miR482b, and miR482e are shown with screenshots from IGV. For display clarity, three biological replicates of each sample were merged. The arrows indicate the sites complementary to miRNAs. The horizontal axes represent the sRNA RPM

$d c l 2 b$ plants through RNA-seq. Compared with the WT, we identified 3435 and 3363 genes that were differentially expressed (DEGs) $(\mid \log 2$ (FoldChange) $\mid>1, p$-value $<0.05)$ in virus-free and virus-infected $d c l 2 b$ mutants, respectively, of which 916 transcripts were overlapped (Fig. 6a middle). In the virus-free $d c l 2 b$ mutant, 1685 transcripts were elevated, whereas 1750 were reduced (Fig. 6a left), and these DEGs might merely represent the DCL2b function under the normal growth condition, whereas DEGs in the virus-infected $d c l 2 b$ would partially result from the virus infection. If we excluded the 916 overlapped transcripts from the total DEGs, the remaining 2447 DEGs were more likely related to defense against viruses. Among the 2447 DEGs, there were 1251 upregulated genes and 1196 downregulated transcripts in the $d c l 2 b$ mutant (Fig. 6a right).

To understand the biological processes associated with $D C L 2 b$-related or virus-affected genes, DEGs were divided into four clusters (upregulated in virus-free $d c l 2 b$ (1), downregulated in virus-free $d c l 2 b$ (2), upregulated in virus-infected $d c l 2 b$ (3), and downregulated in virusinfected $d c l 2 b(4))$, and then they were subjected to Gene Ontology (GO) analysis separately (Table S2). For Cluster 1 , the genes involved in the mitochondrial RNA metabolic process and the defense response were highly enriched (Fig. 6b). We tested the expression of nine genes in the mitochondrial RNA metabolic process pathway and found that all of them belonged to the pentatricopeptide repeat (PPR) protein family (Fig. 6c). These PPR genes have a range of essential functions in post-transcriptional processes within the mitochondria and chloroplasts ${ }^{36}$. Our GO analysis showed that Cluster 2 genes were highly enriched in a hormone metabolism pathway (Fig. 6d). We examined 41 genes that are related to auxin, cytokinin, and other hormones such as gibberellic acid (GA) and found that they were reduced significantly (Fig. 6e). 
a

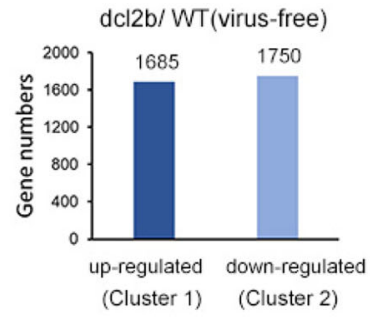

b

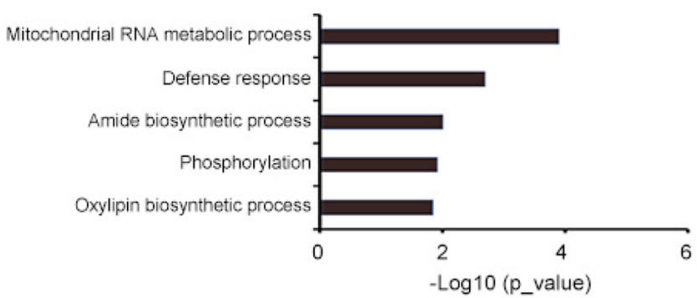

d

Cluster 2

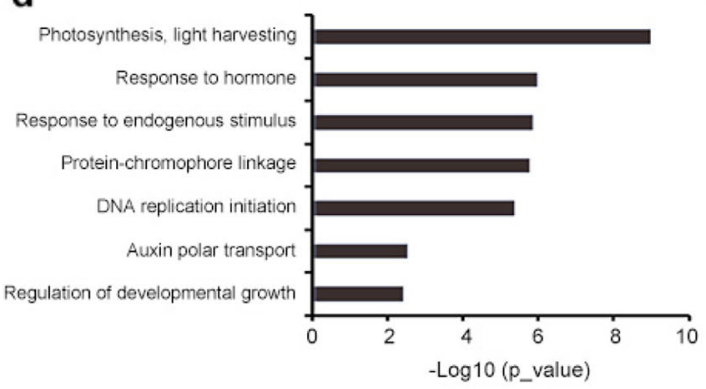

f

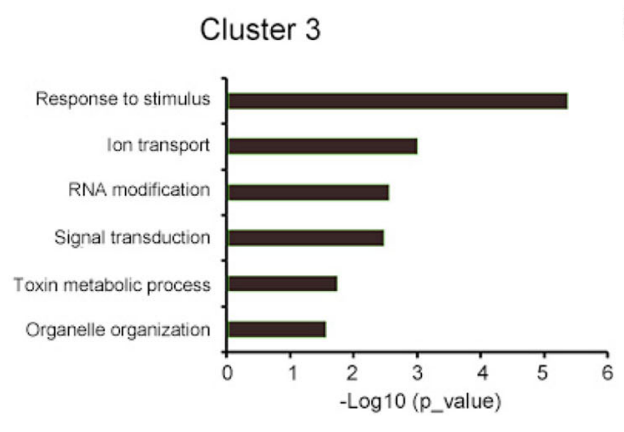

h

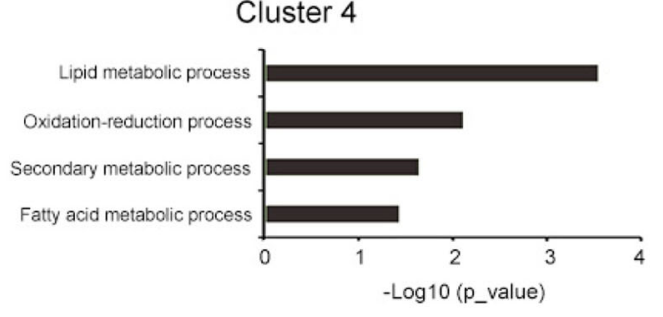

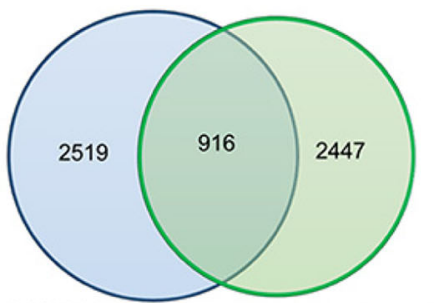

$d c / 2 b$ (virus-infected)

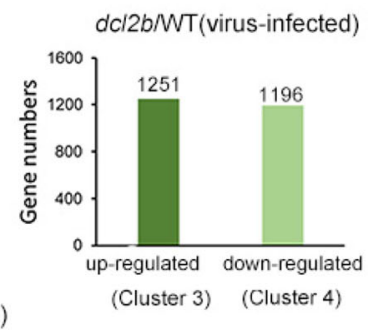

C

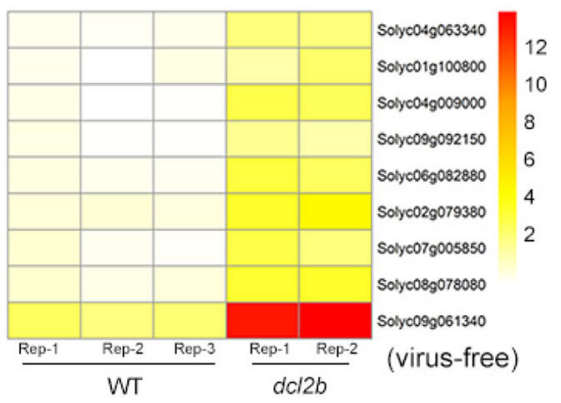

e

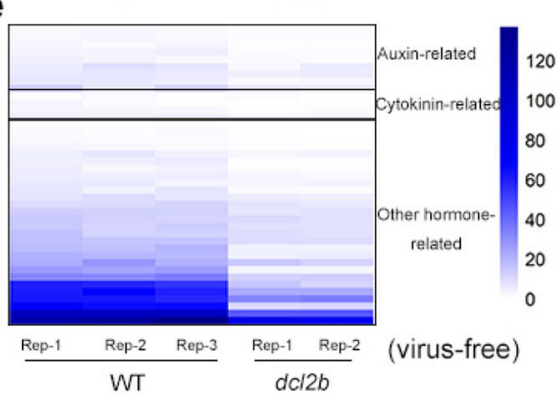

g

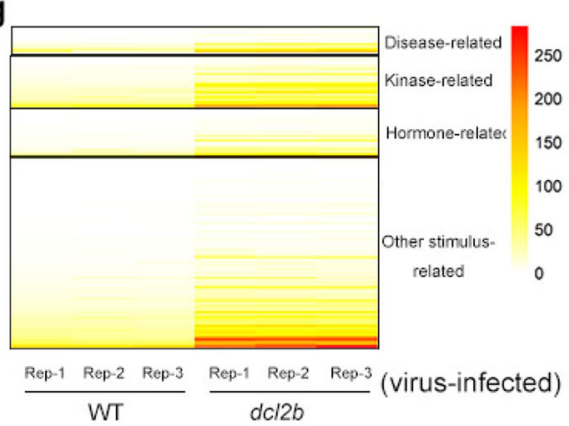

i

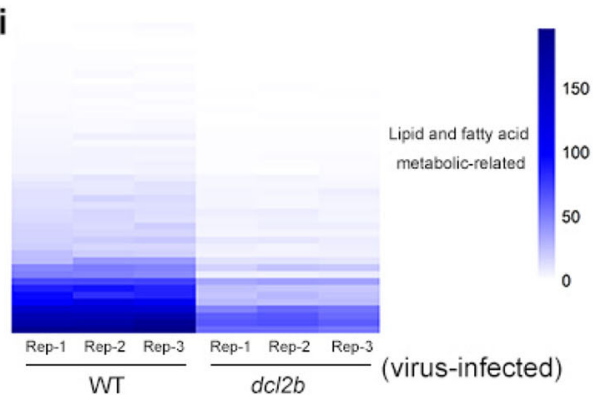

Fig. 6 Gene Ontology enrichment analysis for the differentially expressed genes. a Venn diagrams for numbers of DEGs in the $d c / 2 b$ mutant. The left panel shows up- (Cluster 1) and downregulated (Cluster 2) genes in virus-free $d c / 2 b$. The right panel shows up- (Cluster 3) and downregulated (Cluster 4) genes in virus-infected $d c / 2 b$ after excluding the overlapped 916 transcripts. $\mathbf{b}, \mathbf{d}, \mathbf{f}, \mathbf{h} \mathrm{GO}$ enrichment analysis of Cluster 1-Cluster 4 genes. c, e, $\mathbf{g}$, i Heat map of genes enriched during the mitochondrial RNA metabolic process, hormone signaling, response to stimulus, and lipid and fatty acid metabolism pathways, respectively 
Next, we performed a GO enrichment analysis of virusinduced genes in Cluster 3. As shown in Fig. 6f, genes associated with responses to stimuli were highly enriched. Of all 145 transcripts, 12, 27, and 22 genes belonged to disease-, kinase-, and, hormone-related categories, respectively (Fig. 6g), suggesting that these genes could play an important role in responding to ToMV infection. For Cluster 4, plenty of DEGs were related to the lipid and fatty acid metabolic pathway (Fig. 6h). When viruses invade a cell to complete the replicative cycle, they will express their own proteins and co-opt host cell factors for multiplication, including lipids ${ }^{37}$. The initial steps include the attachment to a specific receptor, in some cases a specific lipid. The replication of the viral genome then takes place, either in association with cellular membranes or other lipid structures. Next, new virus genomes are enclosed inside synthesized viral particles, in which many lipids also play an important role ${ }^{38}$. Since host lipids are essential for multiple steps of the viral replication cycle, plants can use different strategies to interfere with viral infection. One of the strategies is to reduce the lipid metabolism and biogenesis, which might explain why the lipid-related genes were downregulated in $d c l 2 b$ (Fig. 6i).

\section{Discussion}

Here, we systematically studied the function of tomato $D C L 2 b$. Generally, $D C L 2 b$ protein might have a role comparable to that of Arabidopsis DCL2 in producing 22nt sec-siRNAs. However, this very protein also has some unique functions in the biogenesis of 22-nt miRNAs, which in turn cause the production of sec-siRNAs. This function has typically been assigned to DCL1 in Arabidopsis. In addition, the tomato $D C L 2 b$ appears to play a more important role than Arabidopsis DCL2 in the defense against viruses.

\section{Tomato $D C L 2 b$ was responsible for sec-siRNA biogenesis}

In Arabidopsis, 22-nt sRNAs are the key determinant of sec-siRNA triggers ${ }^{16}$. DCL2 usually generates 22 -nt siRNAs from perfectly duplexed precursors ${ }^{21}$. For 22ntmiRNA, its precursor contains a bulge, so DCL1 generates a 22-nt miRNA and a 21-nt miRNA*16,39. In our research, we found that tomato $D C L 2 b$ was required for the biogenesis of 22-nt siRNAs, which served as triggers to generate sec-siRNAs (Fig. 3d and Table S1). Notably, the level of 22-nt miR6026 decreased significantly in the $d c l 2 b$ mutant (Fig. 4a, b). In a previous report, miR6026 was not processed by tomato $D C L 1^{7}$. Moreover, its precursor does not contain asymmetric bulges (miRBase: http://www. mirbase.org/), raising the possibility that miR6026 is $D C L 2 b$-dependent. Additionally, miR6026 was predicted to target tomato $D C L 2 a, 2 b$, and $2 \mathrm{~d}^{7}$. In our study, we also discovered that miR6026 could trigger sec-siRNAs from these three DCL2 transcripts (Fig. 5a), suggesting that there may be a feedback regulation mechanism between $D C L 2 b$ and miR6026.

When tomato plants were infected by ToMV, a large number of 21-nt sRNAs were markedly enhanced (Fig. 3b, c). The expression levels of $D C L 2 b$ accumulated as well, both in the WT and $d c l 2 b$ mutant (Figure S4c and S6). The above feedback loop was destroyed and miR6026 was not decreased in the virus-infected $d c l 2 b$ mutant (Fig. $4 \mathrm{~b}$ ). Other 22-nt miRNAs showed downregulation (Fig. 4c). $D C L 2 b$ might not participate in their processing directly but could influence their expressions when the tomato was infected by a virus. These 22-nt miRNAs acted as secsiRNA triggers, and all of them targeted disease-related genes (Fig. 5c). In the tomato, Tobacco mosaic virus resistance-1 $(T m-1)$ encodes a protein that can bind to ToMV replication proteins and inhibit its replication ${ }^{40,41}$. Due to its low expression $(\mathrm{RPM}<1)$, we could not determine if Tm-1 serves as a sec-siRNA precursor. However, when we calculated the precursors that generated less sec-siRNAs in $d c l 2 b$, we found 197 transcripts, and one-third of them belonged to the "disease-resistant" category (Fig. 4e and Table S1), suggesting that the tomato $D C L 2 b$ appears to regulate disease-related genes by processing sec-siRNAs while combating ToMV.

\section{Tomato $D C L 2 b$ played a key role in defending against ToMV}

In Arabidopsis, DCL4 and DCL2 act hierarchically in antiviral resistance ${ }^{24}$. DCL4 is considered the leader and is primarily responsible for the processing of 21-nt vsiRNAs from RNA viruses ${ }^{20}$. Only when $D C L 4$ is absent or its activity is suppressed by viruses does $D C L 2$ produce 22-nt vsiRNAs, serving as a backup to functionally compensate for $D C L 4^{19,42}$. Turnip crinkle virus (TCV) is an example because this virus encodes a suppressor P38 that specifically inhibits DCL4 activity. In this scenario, DCL2 contributes to a majority of vsiRNAs ${ }^{19}$. For other viruses such as Potato virus $\mathrm{X}$ (PVX), DCL4 alone is sufficient to inhibit PVX accumulation. The infected Arabidopsis dcl2 mutant does not show viral symptoms ${ }^{43}$. During Turnip mosaic virus (TuMV) infection in Arabidopsis, DCL4-dependent siRNAs are necessary to prevent initial infections whereas $D C L 2$ is neither necessary nor sufficient to limit infections ${ }^{20}$. In our study, when the tomato $D C L 2 b$ itself was knocked out, the plants showed severe developmental defects both in natural and manual ToMV infection environments (Fig. 1a, S4a and S4d). This scenario has not been observed with Arabidopsis $D C L 2$, implying that the tomato $D C L 2 b$ has more important roles in virus defense compared to its Arabidopsis counterpart. When the tomato was infected with ToMV, the DCL4 expression remained unchanged (Figure S4c and S6). The 21-nt vsiRNAs and tomato endogenous sRNA also did not decrease (Fig. 2b), suggesting that DCL4 was still functional after antiviral silencing. The ToMV virulence in tomatoes is 
very strong, and plants need both $D C L 2 b$ and $D C L 4$ to combat the virus. Together, these results indicate that plants might be regulated by unique mechanisms during different species-virus interaction.

\section{Materials and methods}

Plant materials and growth conditions

The tomato wild type (cv. Ailsa Craig) and mutants (with AC background) were planted in commercial tomato cultivation soil. All the plants were grown in two greenhouses under the same conditions, $20-25^{\circ} \mathrm{C}$ under a $16 \mathrm{~h}$ light $/ 8 \mathrm{~h}$ dark cycle.

\section{CRISPR/Cas9 gene knockout and mutation analysis}

Four sgRNAs targeting four exons of $D C L 2 b$ were designed using the online tool CRISPR-GE (http://skl. scau.edu.cn/home/). These 20 bp oligos were cloned into AtU3d, AtU3b, AtU6-1, and AuU6-29 vectors, respectively. The above sgRNA expression cassettes were assembled into pYLCRISPR/Cas9-Ubi-H binary plasmid by Golden Gate ligation ${ }^{44}$. Tomato tissue culture was performed according to the established protocol using the Agrobacterium infection method ${ }^{45}$. For the mutation analysis, genomic DNA was extracted from young tomato leaves using a Plant Genomic DNA Kit (Tiangen, China). The DNA was used as a template to amplify the $D C L 2 b$ fragment using PCR. The fragments were then sent out for sequencing. The primers used in the vector construction and mutation analyses are listed in Table S3.

\section{Virus inoculation procedures}

Viral inoculations were performed as described ${ }^{46}$. The tomato plants were infected at 2-, 3-, and 4-week stages and the inoculated plants were placed in the incubator at $22^{\circ} \mathrm{C}$.

\section{High-throughput sequencing of RNAs and sRNAs}

The total RNA samples were prepared from WT and $D C L 2 b$ mutant adult leaves using TRIzol reagent (Invitrogen, USA). Paired-end mRNA libraries were generated using NEBNext ${ }^{\oplus}$ Ultra $^{\mathrm{TM}}$ RNA Library Prep Kit for Illumina (NEB, USA) according to the manufacturer's recommendations and were sequenced on an Illumina HiSeq 4000 platform; $150 \mathrm{bp}$ reads were generated. The quality of clean reads was checked using the FastQC program (v0.11.3). Then, Fastq data were aligned to the tomato genome (SGN release version SL3.0) using TopHat (v2.1.0). These mapped reads were counted by HTSeq (v0.6.1) and differential expression analysis was performed using the DESeq2 package. sRNA libraries were prepared using NEBNext Multiplex Small RNA Library Prep Set for Illumina (NEB, USA.) and sequenced on an Illumina HiSeq2500 platform, and $50 \mathrm{bp}$ single-end reads were generated. The quality of clean reads was also checked by FastQC. Subsequently, FASTA data were aligned to the virus genomes from NCBI (ftp://ftp.ncbi.nlm.nih.gov/refseq/release/viral/) using Bowtie (v1.1.2). The unmapped reads were then aligned with the tomato genome. A differential expression analysis was processed with an in-house python script.

RNA extraction, real-time PCR analysis, and northern blot

The total RNA was extracted with TRIzol reagent from the tomato leaves (Invitrogen, USA). For reverse transcription, $1 \mu \mathrm{g}$ of RNA and oligo dT primers were used to synthesize cDNA using a TranScript One-Step gDNA Removal and cDNA Synthesis SuperMix kit (Trans, China). Real-time PCR was then performed on a CFX96 Real-Time PCR Detection System (Bio-Rad, USA) with SYBR Green PCR Master Mix (Trans, China). Actin was used as an internal control. Each experiment included three independent biological repeats and three technical replicates.

For the ToMV northern blot, $10 \mu \mathrm{g}$ of total RNA was used in each lane. Hybridizations were performed with biotin-labeled probes complementary to the ToMV sequence. Loading RNA served as the control. For the small RNA northern blot, $10 \mu \mathrm{g}$ of total RNA was used in each lane. Hybridizations were performed with $32^{\mathrm{P}}$-radiolabeled probes complementary to miR159, miR390a, and miR6022. U6 served as the loading control ${ }^{6}$. The primers and probes used in the real-time PCR analysis and northern blot are listed in Table S3.

\section{Imaging SEM}

For SEM analysis, leaf samples were first fixed in $2.5 \%$ glutaraldehyde buffer with $0.1 \mathrm{~mol} / \mathrm{L}$ sodium phosphate $(\mathrm{pH}$ 7.2) for $2 \mathrm{~h}$. After the samples were dehydrated with serial ethanol washes, they were dried with a critical point dryer and then coated with gold particles. The samples were examined with an SEM (HITACHI S-3400N, Japan) ${ }^{47}$.

\section{Gene Ontology analysis}

Gene Ontology enrichment analysis was performed using Gene Ontology Consortium online tools ${ }^{48}$. The genes were calculated by PANTHER overrepresentation method. Binomial tests with Bonferroni correction were used to calculate the $p$-values. Terms with $p$-values $<0.05$ were considered to be enriched.

\section{Phylogenetic analysis}

Protein sequences from 34 Tobamovirus were aligned using the ClustalX 2.1 with default parameters ${ }^{49}$ and a phylogenetic tree was constructed through MEGA6.06 by neighbor-joining method with 1000 bootstrap replicates and visualized with the online tool Evolview ${ }^{50}$.

\section{Statistical analysis}

SPSS statistics (v19.0) was used for the statistical analysis. The statistical significance was computed using Student's $t$ test. Significant differences $(p<0.05)$ were 
indicated by asterisks. All data were presented as the means \pm standard errors (SEs).

\section{Data availability}

Raw data from RNA-seq and sRNA-seq have been submitted to the Sequence Read Archive (SRA) at NCBI (http://www.ncbi.nlm.nih.gov/sra/) under the accession number SRP136048.

\section{Acknowledgements}

We would like to thank Dr Yaoguang Liu (South China Agriculture University) for providing the pYLCRISPR/Cas9 system vectors. This research was supported by grants from the National Natural Science Foundation of China (31471921, 91540118,31622050 , and 31672208) to H.Z. T.W. was supported by a fellowship from the Chinese Scholarship Council.

\section{Author details}

${ }^{1}$ College of Food Science and Nutritional Engineering, China Agricultural University, 100083 Beijing, China. ${ }^{2}$ Key Laboratory of Horticultural Plant Biology, Ministry of Education, College of Horticulture and Forestry Sciences, Huazhong Agricultural University, 430070 Wuhan, China. ${ }^{3}$ State Key Laboratory of Protein and Plant Gene Research, College of Life Sciences, Peking University, 100871 Beijing, China. ${ }^{4}$ State Key Laboratory of Plant Genomics, Institute of Genetics and Developmental Biology, Chinese Academy of Sciences, 100101 Beijing, China. ${ }^{5}$ State Key Laboratory of Agro-Biotechnology, College of Biological Sciences, China Agricultural University, 100193 Beijing, China. ${ }^{6}$ National Maize Improvement Center, China Agricultural University, 100193 Beijing, China. ${ }^{7}$ Institute of Environment and Sustainable Development in Agriculture, Chinese Academy of Agricultural Sciences, 100081 Beijing, China

\section{Author contributions}

H.Z. and T.W. designed the experiments; T.W. performed most of the experiments; Z.D. and X.Z. performed the ToMV infections; T.W., H.W., Y.W., X.L., S.L. and F.X. analyzed the data; T.L., D.F., B.Z. and Y.L. provided materials and intellectual input for the work; and T.W. and H.Z. wrote the article.

\section{Conflict of interest}

The authors declare that they have no conflict of interest.

\section{Publisher's note}

Springer Nature remains neutral with regard to jurisdictional claims in published maps and institutional affiliations.

Supplementary Information accompanies this paper at (https://doi.org/ 10.1038/s41438-018-0073-7).

Received: 18 July 2018 Accepted: 27 July 2018

Published online: 01 September 2018

\section{References}

1. Achkar, N. P., Cambiagno, D. A. \& Manavella, P. A. miRNA biogenesis: a dynamic pathway. Trends Plant. Sci. 21, 1034-1044 (2016).

2. Li, S., Castillo-Gonzalez, C., Yu, B. \& Zhang, X. The functions of plant small RNAs in development and in stress responses. Plant J. 90, 654-670 (2017).

3. Holoch, D. \& Moazed, D. RNA-mediated epigenetic regulation of gene expression. Nat. Rev. Genet. 16, 71-84 (2015)

4. Blevins, T. et al. Identification of Pol IV and RDR2-dependent precursors of 24 nt siRNAs guiding de novo DNA methylation in Arabidopsis. elife 4, e9591 (2015).

5. Fukudome, A. \& Fukuhara, T. Plant dicer-like proteins: double-stranded RNAcleaving enzymes for small RNA biogenesis. J. Plant. Res. 130, 33-44 (2017).

6. Zhu, $\mathrm{H}$. et al. Bidirectional processing of pri-miRNAs with branched terminal loops by Arabidopsis Dicer-like1. Nat. Struct. Mol. Biol. 20, 1106 (2013).
7. Kravchik, M. et al. Global and local perturbation of the tomato microRNA pathway by a trans-activated DICER-LIKE 1 mutant. J. Exp. Bot. 65, 725-739 (2014).

8. $\mathrm{Wu}, \mathrm{Y}$. et al. DCL2-and RDR6-dependent transitive silencing of SMXL4 and SMXL5 in Arabidopsis dcl4 mutants causes defective phloem transport and carbohydrate over-accumulation. Plant J. 90, 1064-1078 (2017).

9. Taochy, C. et al. A genetic screen for impaired systemic RNAi highlights the crucial role of DICER-LIKE 2. Plant Physiol. 175, 1424-1437 (2017).

10. Kravchik, M., Damodharan, S., Stav, R. \& Arazi, T. Generation and characterization of a tomato DCL3-silencing mutant. Plant Sci. 221, 81-89 (2014).

11. Yifhar, T. et al. Failure of the tomato trans-acting short interfering RNA program to regulate AUXIN RESPONSE FACTOR3 and ARF4 underlies the wiry leaf syndrome. Plant Cell 24, 3575-3589 (2012).

12. Song, $X$. et al. Roles of $D C L 4$ and $D C L 3 b$ in rice phased small RNA biogenesis. Plant J. 69, 462-474 (2012)

13. Xie, Z. X., Allen, E., Wilken, A. \& Carrington, J. C. DICER-LIKE 4 functions in transacting small interfering RNA biogenesis and vegetative phase change in Arabidopsis thaliana. Proc. Natl Acad. Sci. USA 102, 12984-12989 (2005).

14. Fei, Q., Xia, R. \& Meyers, B. C. Phased, secondary, small interfering RNAs in posttranscriptional regulatory networks. Plant Cell 25, 2400-2415 (2013).

15. Deng, P., Muhammad, S., Cao, M. \& Wu, L. Biogenesis and regulatory hierarchy of phased small interfering RNAs in plants. Plant. Biotechnol. J. 16, 965-975 (2018).

16. Chen, $\mathrm{H}$. et al. 22-nucleotide RNAs trigger secondary siRNA biogenesis in plants. Proc. Natl Acad. Sci. USA 107, 15269-15274 (2010).

17. Xia, R et al. MicroRNA superfamilies descended from miR390 and their roles in secondary small interfering RNA biogenesis in eudicots. Plant Cell 25, 1555-1572 (2013)

18. Axtell, M. J., Jan, C., Rajagopalan, R. \& Bartel, D. P. A two-hit trigger for siRNA biogenesis in plants. Cell 127, 565-577 (2006).

19. Bouche, N., Lauressergues, D., Gasciolli, V. \& Vaucheret, H. An antagonistic function for Arabidopsis DCL2 in development and a new function for DCL4 in generating viral siRNAs. EMBO J. 25, 3347-3356 (2006).

20. Garcia-Ruiz, H. et al. Arabidopsis RNA-dependent RNA polymerases and dicerlike proteins in antiviral defense and small interfering RNA biogenesis during turnip mosaic virus infection. Plant Cell 22, 481-496 (2010)

21. Mlotshwa, S. et al. DICER-LIKE2 plays a primary role in transitive silencing of transgenes in Arabidopsis. PLoS ONE 3, e1755 (2008).

22. Parent, J., Bouteiller, N., Elmayan, T. \& Vaucheret, H. Respective contributions of Arabidopsis DCL2 and DCL4 to RNA silencing. Plant J. 81, 223-232 (2015).

23. Qin, C. et al. Roles of Dicer-Like proteins 2 and 4 in intra- and intercellular antiviral silencing. Plant Physiol. 174, 1067-1081 (2017)

24. Chen, W. et al. A genetic network for systemic RNA silencing in plants. Plant Physiol. 176, 2700-2719 (2018).

25. Bergougnoux, $\mathrm{V}$. The history of tomato: from domestication to biopharming. Biotechnol. Adv. 32, 170-189 (2014).

26. Wang, T. et al. Cloning, identification, and expression analysis of a Dicer-Like gene family from Solanum lycopersicum. Biol. Plant. 60, 410-418 (2016).

27. Xie, X. et al. CRISPR-GE: a convenient software toolkit for CRISPR-based genome editing. Mol. Plant 10, 1246-1249 (2017).

28. Andrade, O., Latorre, B. A. \& EscaffiS, O. Tomato mosaic-virus associated with shoestring sympotem in chilean tomatoes. Plant Dis. 65, 761-762 (1981).

29. Visser, M., Bester, R., Burger, J. T. \& Maree, H. J., Next-generation sequencing for virus detection: covering all the bases. Virol. J. 13, 85 (2016)

30. Kung, $Y$. et al. Genetic analyses of the FRNK motif function of turnip mosaic virus uncover multiple and potentially interactive pathways of crossprotection. Mol. Plant Microbe Interact. 27, 944-955 (2014).

31. Andika, I. B., Jamal, A., Kondo, H. \& Suzuki, N. SAGA complex mediates the transcriptional up-regulation of antiviral RNA silencing. Proc. Natl Acad. Sci. USA 114, E3499-E3506 (2017).

32. Malpica-Lopez, N. et al. Revisiting the roles of tobamovirus replicase complex proteins in viral replication and silencing suppression. Mol. Plant Microbe Interact. 31, 125-144 (2018).

33. Zhu, B. et al. RNA sequencing and functional analysis implicate the regulator role of long non-coding RNAs in tomato fruit ripening. J. Exp. Bot. 66 4483-4495 (2015).

34. Cao, M. et al. Virus infection triggers widespread silencing of host genes by a distinct class of endogenous siRNAs in Arabidopsis. Proc. Natl Acad. Sci. USA 111, 14613-14618 (2014).

35. Vazquez, F. \& Hohn, T. Biogenesis and biological activity of secondary siRNAs in plants. Scientifica 2013, 783253 (2013). 
36. Schmitz-Linneweber, C. \& Small, I. Pentatricopeptide repeat proteins: a socket set for organelle gene expression. Trends Plant. Sci. 13, 663-670 (2008).

37. Nagy, P. D. \& Pogany, J. The dependence of viral RNA replication on co-opted host factors. Nat. Rev. Microbiol. 10, 137-149 (2011).

38. Miguel, M., et al. Lipid involvement in viral infections: present and future perspectives for the design of antiviral strategies. in Lipid Metabolism, pp 291 322 (ed Rodrigo, V. B.) Ch. 13 (InTech, 2013).

39. Li, F. et al. MicroRNA regulation of plant innate immune receptors. Proc. Natl Acad. Sci. USA 109, 1790-1795 (2012).

40. Ishibashi, K. \& Ishikawa, M. The resistance protein Tm-1 inhibits formation of a tomato mosaic virus replication protein-host membrane protein complex. J. Virol. 87, 7933-7939 (2013).

41. Ishibashi, K. et al. Structural basis for the recognition-evasion arms race between Tomato mosaic virus and the resistance gene Tm-1. Proc. Natl Acad. Sci. USA 111, E3486-E3495 (2014).

42. Deleris, A. et al. Hierarchical action and inhibition of plant Dicer-like proteins in antiviral defense. Science 313, 68-71 (2006).

43. Andika, I. B. et al. Differential contributions of plant Dicer-like proteins to antiviral defences against potato virus $X$ in leaves and roots. Plant J. 81, 781-793 (2015).
44. Ma, X. et al. A robust CRISPR/Cas9 system for convenient, high-efficiency multiplex genome editing in monocot and dicot plants. Mol. Plant 8, 1274-1284 (2015).

45. Van Eck, J., Kirk, D. D. \& Walmsley, A. M. Tomato (Lycopersicum esculentum). Methods Mol. Biol. 343, 459-473 (2006).

46. Whitham, S., McCormick, S. \& Baker, B. The N gene of tobacco confers resistance to tobacco mosaic virus in transgenic tomato. Proc. Natl. Acad. Sci. USA 93, 8776-8781 (1996).

47. Li, R. et al. Multiplexed CRISPR/Cas9-mediated metabolic engineering of $\gamma^{-}$ aminobutyric acid levels in Solanum lycopersicum. Plant. Biotechnol. J. 16, 415-427 (2018)

48. Ashburner, M. et al. Gene Ontology: tool for the unification of biology. Nat Genet. 25, 25-29 (2000)

49. Larkin, M. A. et al. Clustal $W$ and clustal $X$ version 2.0. Bioinformatics 23 2947-2948 (2007)

50. He, Z. et al. Evolviewv2: an online visualization and management tool for customized and annotated phylogenetic trees. Nucleic Acids Res. 44, W236-W241 (2016). 